\title{
CONF-961123--6 \\ Integrated Constant-Fraction Discriminator Shaping Techniques for the PHENIX Lead-Scintillator Calorimeter
}

\author{
R. Gentry Jackson, T. Vaughn Blalock \\ University of Tennessee, Knoxville \\ Michael L. Simpson, Alan L. Wintenberg and Glenn R. Young \\ Oak Ridge National Laboratory
}

\section{DISTRIBUTION OF THIS DOCUMENT IS UNUUATEO<smiles>[C]1C#C1</smiles>

*Research sponsored by the U.S. Department of Energy and performed at Oak Ridge National Laboratory, managed by Lockheed Martin Energy Research Corporation for the U.S. Department of Energy under Contract No. DE-AC05-96OR22464. 


\section{DISCLAIMER}

This report was prepared as an account of work sponsored by an agency of the United States Government. Neither the United States Government nor any agency thereof, nor any of their employees, makes any warranty, express or implied, or assumes any legal liability or responsibility for the accuracy, completeness, or usefulness of any information, apparatus, product, or process disclosed, or represents that its use would not infringe privately owned rights. Reference herein to any specific commercial product, process, or service by trade name, trademark, manufacturer, or otherwise does not necessarily constitute or imply its endorsement, recommendation, or favoring by the United States Government or any agency thereof. The views and opinions of authors expressed herein do not necessarily state or reflect those of the United States Government or any agency thereof. 


\section{DISCLAIMER}

Portions of this document may be illegible in electronic image products. Images are produced from the best available original document. 


\title{
Integrated Constant-Fraction Discriminator Shaping Techniques for the PHENIX Lead-Scintillator Calorimeter ${ }^{1}$
}

\author{
R. Gentry Jackson, T. Vaughn Blalock \\ University of Tennessee, Knoxville \\ Michael L. Simpson, Alan L. Wintenberg, and Glenn R. Young \\ Oak Ridge National Laboratory
}

\begin{abstract}
The suitability of several on-chip constant-fraction discriminator (CFD) shaping methods for use in the multichannel PHENIX Lead-Scintillator detector has been investigated. Three CFD circuits utilizing a distributed R-C delay-line, a lumped-element R-C delay-line and the Nowlin shaping method have been realized in a standard $1.2-\mu \mathrm{m} n-$ well CMOS process. A CFD using ideal delay-line shaping was also studied for comparison. Time walk for $5 \mathrm{~ns}$ risetime input signals over a dynamic range of $-2 \mathrm{~V}$ to $-20 \mathrm{mV}$ was less than $\pm 175 \mathrm{ps}, \pm 150 \mathrm{ps}, \pm 150$, and $\pm 185 \mathrm{ps}$ while worst case rms timing jitter measured $85 \mathrm{ps}, 90 \mathrm{ps}, 100 \mathrm{ps}$, and 65 ps, respectively, for the four methods mentioned above. Area requirements for the three candidate methods tested including the fraction circuit were $172 \mu \times 70 \mu, 160 \mu \times 65 \mu, 179 \mu \times$ $53 \mu$, respectively. The fraction circuit area for the external delay-line circuit was $67 \mu \times 65 \mu$. Each shaping method studied consumed no power from the dc supply.
\end{abstract}

\section{INTRODUCTION}

The PHENIX Lead-Scintillator $(\mathrm{PbSc})$ system will consist of $\sim 15,500$ channels comprised of a detector with alternating layers of lead and scintillating tiles, an FEU115M photomultiplier tube (PMT), a PMT base, and integrated readout electronics. This system will be capable of providing energy, trigger summation, and timing measurements. The timing measurements are required for separation of photons and particles with mass.

Readout electronics for this system will be a low-power integrated circuit (IC) system comprised of a two-chip set realized in CMOS technology. The first chip will contain four channels of timing discriminators, variable gain amplifiers (VGAs), time-to-amplitude converters (TACs), and tower trigger sum circuits. Programmable digital-to-analog converters (DACs) and calibration circuitry will be provided for biasing and testing, and circuitry is also included to handle serial digital data loaded on-chip. The second chip will contain analog-memory units (AMUs), analog-to-digital converters (ADCs), and buffer register circuits.

\footnotetext{
${ }^{1}$ Research sponsored by the U.S. Department of Energy and performed at the Oak Ridge National Laboratory, managed by Lockheed Martin Energy Research Corporation for the U.S. Department of Energy under contract DE-AC05-96OR22464.
}

The major $\mathrm{PbSc}$ timing requirement is sub-nanosecond resolution over an input signal amplitude dynamic range of 100:1. Constant-fraction discrimination techniques will be employed to meet the this goal. In addition to uncertainties inherent to the detector itself, time walk and timing jitter introduced by the timing measurement circuitry affect the timing resolution. These factors are discussed below and analyzed in this work for several integratable constant-fraction discriminator (CFD) shaping methods.

\section{TIming MEasurement UnCERTAINTIES}

Time walk is the variation in time of a discriminator's output due to input signal amplitude changes. Time walk is also influenced by many factors including signal risetime variations or the non-ideal discriminator properties. The shaped signal under-drive, over-drive, and zero-crossing slope properties are determined by the shaping network and have been shown to affect timing performance [1].

Timing jitter is caused by electronic noise in a system and generates variations in repeated timing measurements. Jitter is given by the triangle rule as [2]

$$
\sigma_{\mathrm{t}}=\left(\sigma_{\mathrm{v}} / \mathrm{K}\right)+\delta
$$

where $\sigma_{\mathrm{t}}$ is the rms timing jitter, $\sigma_{v}$ is the rms noise voltage at the output, $\mathrm{K}$ is the shaped signal slope through the zerocrossing, and $\delta$ is jitter introduced by signal-shape variations from the detector. To minimize jitter, the rms noise must be minimized while signal slope is maximized.

\section{CFD SHAPING METHODS}

The constant-fraction discrimination shaping network converts the unipolar input signal into a bipolar signal with the zero-crossing used as a precise timing marker. The classical approach to CFD shaping methods is illustrated in Figure 1. A bipolar signal is generated by the subtraction of a delayed copy

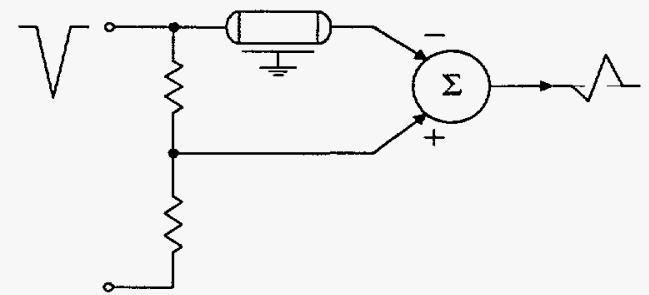

Figure 1: Illustration of the traditional CFD shaping method. 
of the original input from a fraction of the original input signal. The delay time and fraction can be approximated for input signals with nearly linear edges based on the relationship given by [2]

$$
\operatorname{td}=\operatorname{tr}(1-f)
$$

where td is the delay, tr is the input signal risetime, and $\mathrm{f}$ is the fraction. This shaping technique generates zero-crossing times independent of changes in signal amplitude. Typically, the delay signal for shaping has been generated with a length of coaxial cable. Although this technique is optimal in terms of shaped signal characteristics, the cost and space required for the delay cable prove prohibitive for a multichannel detector design. For this reason, much work has been devoted to the realization of integratable CFD shaping methods.

Recent developments in integrated timing pick-off circuits have included a CMOS constant-fraction discriminator using an external delay-line [3], lumped-element shaping methods [4-10], and fully integrated CMOS CFDs [5-8]. These fully integrated CFDs have allowed the realization of large, multichannel time-interval measurement circuits for physics experiments and medical imaging applications. In this work, various shaping methods have been compared on the basis of area, power, timing walk and rms timing jitter.

Several options exist for a fully integrated shaping method solution. The simplest form of shaping is the C-R differentiator $[6,8]$ which requires no fraction circuit and is shown in Figure 2a. Since signals that peak possess a sign change in the derivative, the C-R differentiator will produce a bipolar signal. With this method, the slope through the zerocrossing and overdrive are dependent on the trailing edge of the input signal. This can introduce more timing jitter into the measurement in the presence of white noise and possibly increase walk. However, the high-pass nature of the differentiation could provide lower timing jitter in the presence of high flicker noise. Due to the longer trailing-edge produced by the PbSc PMTs, the simple C-R differentiation shaping will not be considered in this work.

Figure $2 \mathrm{~b}$ shows the lumped-element R-C shaping network [4]. This method generates a delay signal with a single R-C low-pass filter. Two undesirable characteristics of this network associated with low-pass filtering are signal amplitude and slope degradation.

The distributed R-C delay-line shaping network [7] shown in Figure $2 c$ is also a candidate for this application. In this method, the delay signal for shaping is realized with a serpentine layer of polysilicon over a second grounded polysilicon layer. The polysilicon line is a lossy transmission line with delay and dispersion.

The Nowlin method $[8,9]$ is the last shaping technique investigated in this work. This method utilizes the C-R differentiation with a fraction circuit as seen in Figure $2 \mathrm{~d}$. The attenuated signal is subtracted from the differentiated signal to produce the shaped signal. This shaping circuit can be optimized to produce risetime and amplitude compensated timing measurement, although risetime variations are not expected in this application.

Binkley [5] reported a shaping method using a 4-stage Gaussian filter to generate delay. The die area required to realized this 4-pole network is relatively large and is not desirable for multichannel detector applications. The Binkley method will not be considered further in this work.

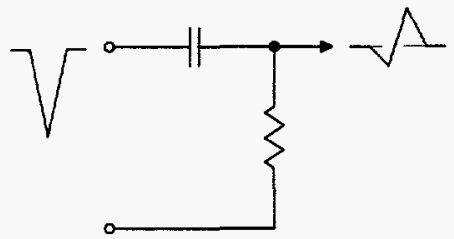

(a)

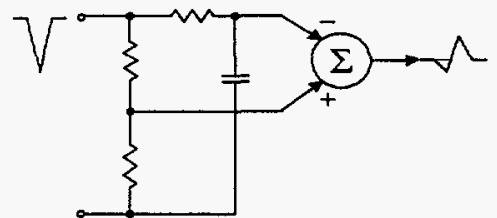

(b)

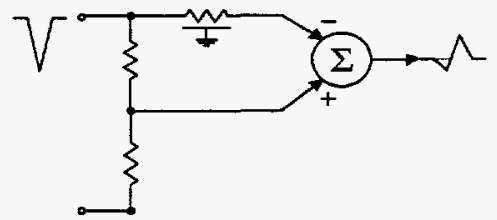

(c)

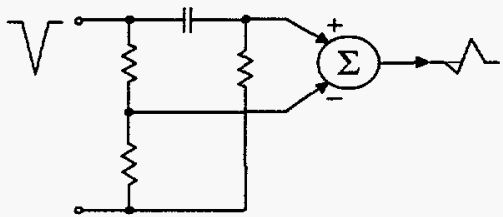

(d)

Figure 2: Illustration of four CFD shaping methods. (a) C-R differentiator. (b) Lumped-Element R-C. (c) Distributed R-C delayline. (c) Nowlin shaping method.

\section{SIMULATION DATA RESULTS}

Candidate shaping methods selected for this work were the lumped-element R-C filter, the distributed R-C delay-line, and the Nowlin method. The ideal delay-line shaping was also studied for comparison. The performance of these shaping methods was analyzed with a $-20 \mathrm{mV}$ pseudo-gaussian input signal approximating rise- and falltimes of the PHENIX PbSc PMT. The CFD possessed six amplifiers in the zero-crossing comparator (ZCC) each with a gain of $\sim 2.3 \mathrm{~V} / \mathrm{V}$ and a bandwidth of $\sim 110 \mathrm{MHz}$. For simulation purposes, the discriminator was approximated by cascaded stages of amplification and low-pass filtering. Noise analysis was performed with an input white noise source of $\sim 400 \mathrm{nV} / \mathrm{JHz}$ at the shaping network input. The large value of this noise source was selected to isolate the jitter caused by input noise from the jitter generated by the inherent noise of the shaping network and the discriminator. 
Shaped signal zero-crossing slope, total rms output noise, and rms timing jitter were also compared for each method. The three methods that generated a delay copy of the original signal were compared based on the peak position delay times in Figures 3 - 5. Since the Nowlin method does not produce a delayed copy of the original as the other methods, it was compared to the other methods on the basis of the C-R differentiator time constant.

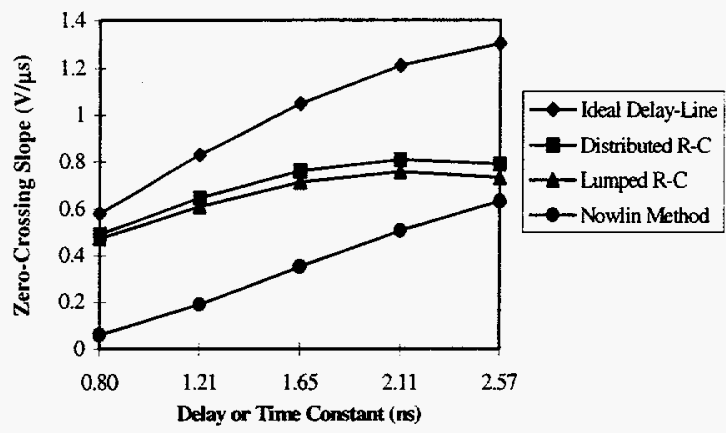

Figure 3: Shaped signal zero-crossing slope.

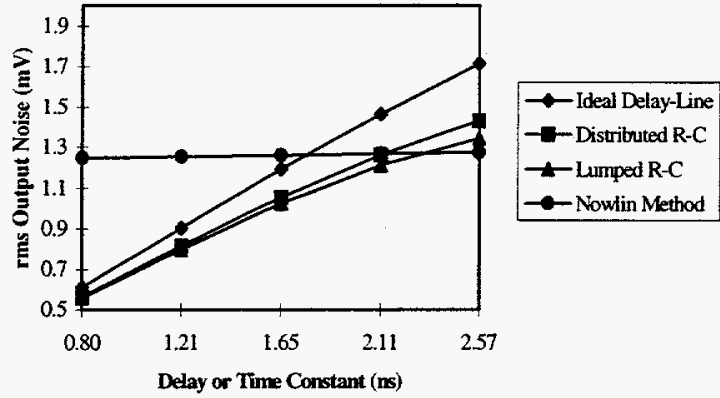

Figure 4: Shaping network total rms output noise.

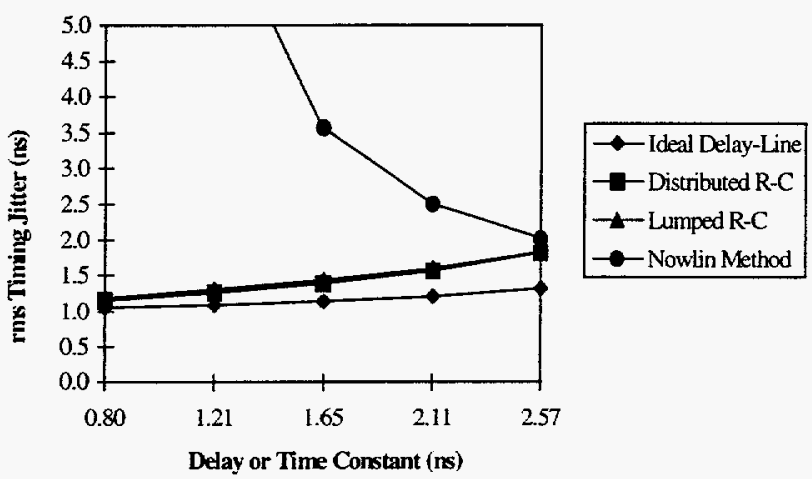

Figure 5: Shaping network rms timing jitter.

With no signal degradation in the delayed copy, the ideal delay-line exhibits the largest slope through the zero-crossing. Also, the total rms output noise of the ideal delay-line is higher due to the absence of filtering seen in the other methods. Signal degradation in the delayed signal of the distributed R-C delay-line and the lumped-element $\mathrm{R}-\mathrm{C}$ shaping cause the slope roll-off at longer delays. The Nowlin method exhibits worse slope through the zero-crossing at lower C-R time constants due to severe amplitude attenuation. The relatively flat rms output noise with C-R time constant seen in Figure 4 for the Nowlin method was due to discriminator frequency response dominating the noise transfer function.

Time walk simulations were performed over a 100:1 input amplitude dynamic range with a maximum input signal of - 2 V. Figure 6 shows the walk for each method with increasing delay time or C-R time constant. Each shaping method exhibited lower time walk with increasing delay time or C-R time constant. A delay time corresponding to $\sim 1.21 \mathrm{~ns}$ was chosen for fabrication for the methods that produce a delayed copy of the original input signal. The Nowlin shaping method time constant chosen for fabrication was $\sim 2.3$ ns. Figure 7 shows the simulated time walk curves for each shaping method chosen over a 100:1 input amplitude dynamic range.

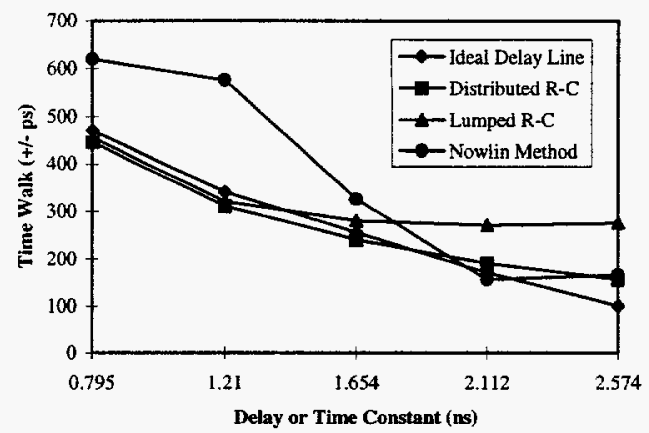

Figure 6: Time walk simulated data for shaping methods.

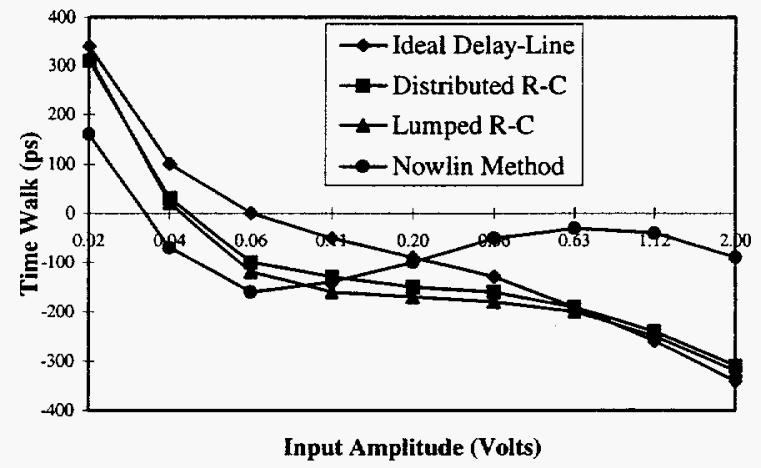

Figure 7: Time walk simulated over a 100:1 input amplitude dynamic range for each method fabricated in $5 \mathrm{~dB}$ steps.

\section{RESULTS}

Each shaping method was fabricated in the Orbit Semiconductor $1.2-\mu \mathrm{m} n$-well CMOS process. The different shaping methods were tested with an input signal possessing $5 \mathrm{~ns}$ and $-15 \mathrm{~ns}$ rise- and fallimes, respectively. Time walk measurements over a 100:1 input amplitude dynamic range ( $2 \mathrm{~V}$ to $-20 \mathrm{mV}$ ) were taken for each method. Table 1 gives the time walk for ten ICs tested. Figure 8 shows the best, typical, and worst case time walk plotted around a mean of zero and in $5 \mathrm{~dB}$ steps along the $\mathrm{x}$-axis. Lumped-element R-C and Nowlin method shaping possessed typical time walk of \pm 150 ps while distributed R-C delay-line and ideal delay-line exhibited time walk of \pm 175 ps and \pm 185 ps, respectively. 
Timing jitter due to inherent CFD circuit noise was also determined for each shaping method channel for the minimum input signal and listed in Table 2. Figure 9 shows the rms timing jitter measurement from a typical CFD channel of each shaping method as a function of input amplitude. The ideal delay-line exhibited the best rms timing jitter performance while the Nowlin shaping method exhibited the worst rms timing jitter performance due to inherent CFD circuit noise for minimum input signals.

Table 1.

Table of time walk measurements for each IC tested.

\begin{tabular}{|c|c|c|c|c|}
\hline & \multicolumn{4}{|c|}{ Time Walk \pm ps $)$} \\
\hline $\begin{array}{c}\text { Chip } \\
\#\end{array}$ & $\begin{array}{c}\text { Ideal } \\
\text { Delay- } \\
\text { Line }\end{array}$ & $\begin{array}{c}\text { Distributed } \\
\text { R-C } \\
\text { Delay-Line }\end{array}$ & $\begin{array}{c}\text { Lumped- } \\
\text { Element } \\
\text { R-C }\end{array}$ & $\begin{array}{c}\text { Nowlin } \\
\text { Method }\end{array}$ \\
\hline 1 & 185 & 150 & 160 & 150 \\
\hline 2 & 300 & 185 & 135 & 135 \\
\hline 4 & 175 & 175 & 135 & NA \\
\hline 5 & 225 & 175 & 150 & 350 \\
\hline 6 & 150 & 175 & 150 & 150 \\
\hline 8 & 175 & 150 & 160 & 160 \\
\hline 9 & 210 & 175 & 135 & 175 \\
\hline 10 & 225 & 200 & 160 & 135 \\
\hline 11 & 185 & 150 & 150 & 150 \\
\hline 12 & 160 & 175 & 175 & 185 \\
\hline
\end{tabular}

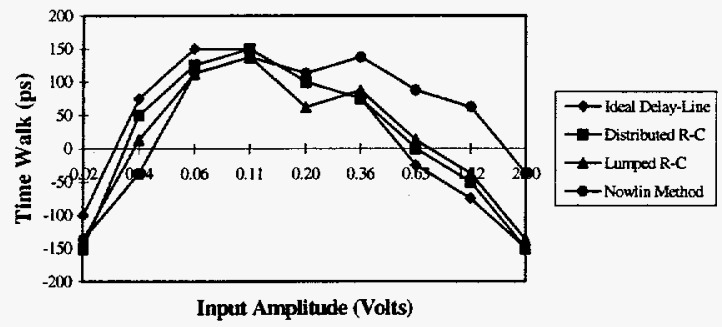

(a)

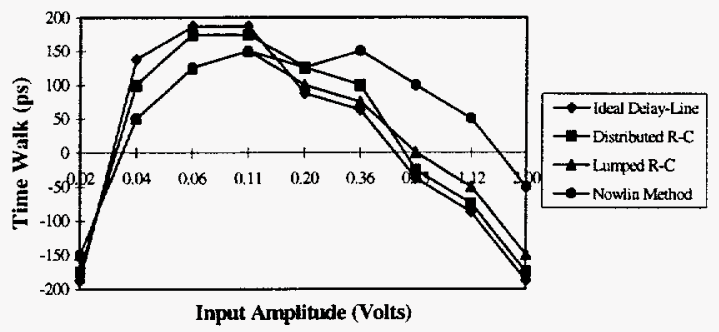

(b)

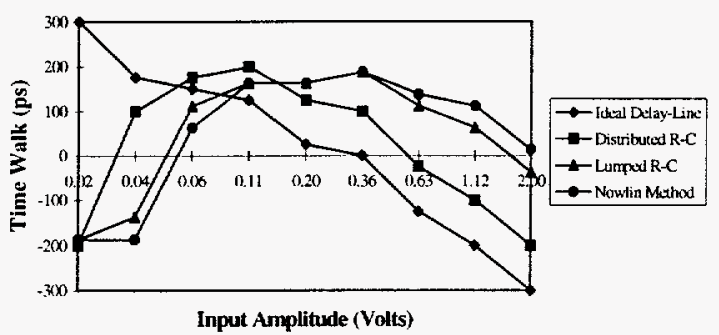

(c)

Figure 8: Time walk for each shaping method. (a) Best case. (b) Typical case. (c) Worst case.
Table 2.

Table of rms timing jitter for $-20 \mathrm{mV}$ peak input.

\begin{tabular}{|c|c|c|c|c|}
\hline & \multicolumn{4}{|c|}{ rms Timing Jitter (ps) } \\
\hline $\begin{array}{c}\text { Chip } \\
\#\end{array}$ & $\begin{array}{c}\text { Ideal } \\
\text { Delay- } \\
\text { Line }\end{array}$ & $\begin{array}{c}\text { Distributed } \\
\text { R-C } \\
\text { Delay-Line }\end{array}$ & $\begin{array}{c}\text { Lumped- } \\
\text { Element } \\
\text { R-C }\end{array}$ & $\begin{array}{c}\text { Nowlin } \\
\text { Method }\end{array}$ \\
\hline 1 & 65 & 85 & 90 & 85 \\
\hline 2 & 100 & 85 & 85 & 90 \\
\hline 4 & 75 & 85 & 90 & NA \\
\hline 5 & 60 & 65 & 85 & 65 \\
\hline 6 & 75 & 65 & 85 & 100 \\
\hline 8 & 85 & 85 & 130 & 100 \\
\hline 9 & 65 & 65 & 85 & 90 \\
\hline 10 & 65 & 85 & 110 & 115 \\
\hline 11 & 65 & 85 & 85 & 100 \\
\hline 12 & 85 & 90 & 85 & 100 \\
\hline
\end{tabular}

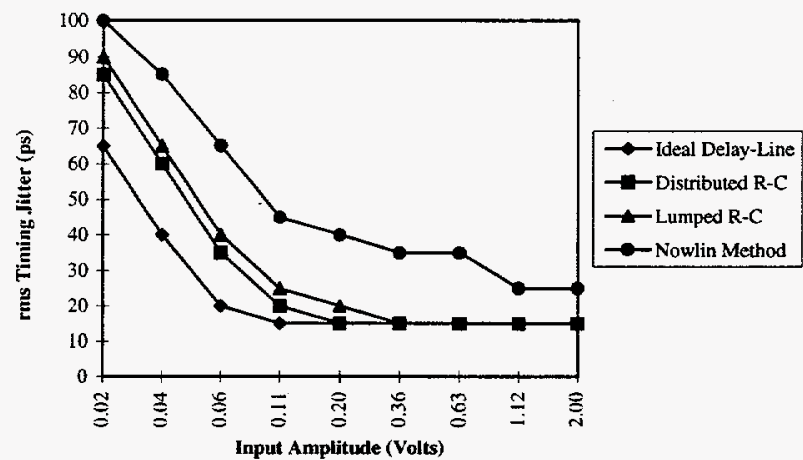

Figure 9: Plot of rms timing jitter for a typical CFD channel of each shaping method with input amplitude.

A second timing jitter measurement was performed to determine the susceptibility of each shaping method to input noise. For a fixed input signal amplitude and shape, the timing jitter due to an input white noise source can be given by

$$
\sigma_{t}^{2}=\int_{0}^{\infty} s_{n}^{2}|J(f)|^{2} d f
$$

where $\sigma_{\mathrm{t}}^{2}$ is the square of the rms timing jitter in $\mathrm{s}^{2}, \mathrm{~S}_{\mathrm{n}}^{2}$ is the power spectral density of the input in $\mathrm{V}^{2} / \mathrm{Hz}$, and $|\mathrm{J}(\mathrm{f})|^{2}$ is the square of the magnitude of the transfer function relating input noise to output timing jitter in $\mathrm{s}^{2} / \mathrm{V}^{2}$. The simulation analysis presented earlier, used a value of $S_{n} \sim 400 \mathrm{nV} / \mathrm{HHz}$, and the input amplitude was $-20 \mathrm{mV}$. To measure this same quantity experimentally, the transfer function J(f) had to be determined.

The transfer function $J(f)$ was determined by introducing a single frequency sine wave and the input pulse in coincidence into the CFD. This measurement was carried out over a large frequency range of sine wave inputs. It can be shown that the transfer function $J(f)$ can be determined by the relationship [12]

$$
J(f)=\frac{J_{\text {SINE_WAVE(p-p) }}(f)}{V_{\text {in( }(p-p)}(f)},
$$


where $J_{\text {SINE_WAVE(p-p) }}(f)$ is the peak-to-peak timing jitter due to the sine wave input with frequency and $V_{i n(-p-p)}(f)$ is the input sine wave amplitude with frequency. For this work the denominator of Equation 4 was held constant over frequency. Substituting Equation 4 into Equation 3 gives the timing jitter to be

$$
\sigma_{t}^{2}=\int_{0}^{\infty} S_{n}^{2}\left|\frac{J_{\text {SINE_WAVE }(p-p)}(f)}{V_{\text {in }(p-p)}(f)}\right|^{2} d f
$$

With Equation 5, the same value of input noise spectral density from simulations could be used to compare measured results. The transfer function of each method was generated as just described and plotted versus frequency in Figure 10. Equation 5 was solved by numerical integration and the results are listed in Table 3. The Nowlin shaping method exhibited much higher rms timing jitter at lower frequencies as expected. The other shaping methods possessed some peaking in the frequency transfer function due to the correlation of the delayed and prompt signal. Simulation data indicated that a higher than expected bandwidth in the CFD ZCC would result in overall decrease in the rms timing jitter for each method. The measured results tend to suggest the ZCC possessed a higher bandwidth than expected. The Nowlin shaping method tended to be the most susceptible to input noise from the detector.

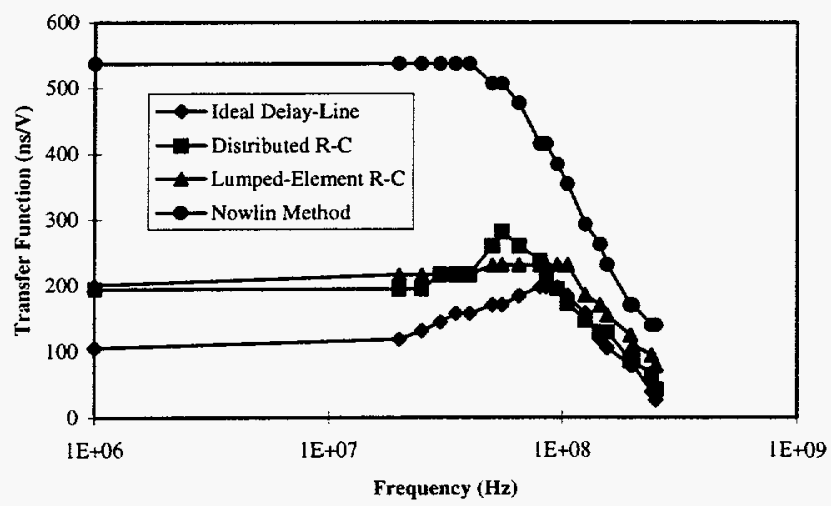

Figure 10: Timing jitter transfer functions showing relative values.

Table 3.

Table comparing simulation predictions to measured results for timing jitter due to an input noise source.

\begin{tabular}{|c|c|c|c|}
\hline Shaping Method & \multicolumn{2}{|c|}{$\begin{array}{c}\text { rms Timing Jitter for } \\
-400 \mathrm{nV} / \mathrm{JHz}\end{array}$} & $\begin{array}{c}\text { Percent } \\
\text { Difference } \\
(\%)\end{array}$ \\
\cline { 2 - 4 } & HSPICE (ns) & Measured (ns) & -19.0 \\
\hline $\begin{array}{c}\text { Ideal Delay-Line } \\
\text { Distributed R-C } \\
\text { Delay-Line }\end{array}$ & 1.05 & 0.85 & -15.1 \\
\hline $\begin{array}{c}\text { Lumped- } \\
\text { Element R-C }\end{array}$ & 1.26 & 1.07 & -12.2 \\
\hline Nowlin Method & 2.27 & 1.15 & -1.0 \\
\hline
\end{tabular}

\section{CONCLUSIONS}

Three constant-fraction discriminator shaping methods realizable in a standard CMOS process have been investigated in this work and compared to an ideal delay-line shaping version. $\mathrm{R}-\mathrm{C}$ delay methods provided better jitter performance than the Nowlin method for the bandwidths obtainable in a $1.2-\mu$ CMOS process. The distributed R-C delay-line shaping offered slightly better performance than the lumped-element $\mathrm{R}-\mathrm{C}$ method and was deemed best for the lead-scintillator CFD.

\section{REFERENCES}

[1] Binkley, D. M., and M. E. Casey, "Performance of Fast Monolithic ECL Voltage Comparators in ConstantFraction Discriminators and other Timing Circuits," IEEE Trans. on Nucl. Sci., Vol. NS-35, No. 1, February 1988, pp. 226-230.

[2] EG\&G ORTEC, "Principles and Applications of Timing Spectroscopy," Application Note AN-42, Oak Ridge, Tennessee.

[3] Binkley, et al., "A Monolithic, $2 \mu \mathrm{m}$ CMOS ConstantFraction Discriminator for Moderate Time Resolution Systems," IEEE Trans. on Nucl. Sci. Vol. NS-38, No. 6, December 1991, pp. 1754-1759.

[4] Turko, B. T. and R. C. Smith, "A Precision Timing Discriminator for High Density Detector Systems," Conference Record of 1991 IEEE Nucl. Sci. Symposium and Medical Imaging Conference. November 1991.

[5] Binkley, D. M., "Performance of Non-Delay-Line Constant-Fraction Discriminator Timing Circuits," IEEE Trans. on Nucl. Sci. Vol. NS-41, No. 4, August 1994, pp. $1169-1175$.

[6] Simpson, M. L., et al, "An Integrated, CMOS, ConstantFraction Timing Discriminator for Multichannel Detector Systems," IEEE Trans on Nucl. Sci. Vol. 42, No. 4, August 1995, pp. 762-766.

[7] Simpson, M. L., et al., "A Monolithic, Constant-Fraction Discriminator Using Distributed R-C Delay-Line Shaping," IEEE Trans on Nucl. Sci., Vol. 43, No. 3, June 1996, pp. 1695-1699.

[8] Simpson, M. L., et al., "An Integrated CMOS Timing Interval Measurement System with Sub-Nanosecond Resolution for the WA-98 Calorimeter," To be published in the Journal of Solid-State Circuits, February 1997.

[9] Nowlin, C. H., U. S. Patent No. 4,443,768 (April 17, 1984).

[10] Nowlin, C. H., "Low-Noise Lumped-Element Timing Filters with Rise-Time Invariant Crossover Times," Rev. of Sci. Instr. Vol. 63, No.4, April 1992, pp. 2322-2326.

[11] Jackson, R. G., Master of Science Thesis, University of Tennessee at Knoxville, December 1996. 